\title{
On the evaluation of both spatial and temporal performance of distributed hydrological models
}

\author{
Tam Nguyen ${ }^{1}$, AN TRAN², Bhumika Uniyal ${ }^{3}$, and Thuc Phan ${ }^{4}$ \\ ${ }^{1}$ Helmholtz-Centre for Environmental Research - UFZ \\ ${ }^{2}$ Thuyloi University \\ ${ }^{3}$ University of Bayreuth \\ ${ }^{4}$ Vietnam Academy of Science and Technology
}

June 17, 2021

\begin{abstract}
Evaluating the spatial and temporal model performance of distributed hydrological models is necessary to ensure that the simulated spatial and temporal patterns are meaningful. In recent years, spatial and temporal remote sensing data have been increasingly used for model performance evaluation. Previous studies, however, have focused on either the temporal or spatial model performance evaluation. In addition, temporal (or spatial) model performance evaluation is often done in a spatially (or temporally) lumped approach. Here, we evaluated (1) the temporal model performance evaluation in a spatially distributed approach (spatiotemporal) and (2) the spatial model performance in a temporally distributed approach (temporospatial) model performance evaluation. This study demonstrated that both spatiotemporal and temporospatial model performance evaluations are necessary since they provide different aspects of the model performance. For example, spatiotemporal model performance evaluation helps in detecting the areas with an issue in the simulated temporal patterns. However, temporospatial model performance evaluation helps in detecting the time with an issue in the simulated spatial patterns. The results also show that an increase in the spatiotemporal model performance will not necessarily lead to an increase in the temporospatial model performance and vice versa, depending on the evaluation statistics. Overall, this study has highlighted the necessity of a joint spatiotemporal and temporospatial model performance evaluation to understand/improve spatial and temporal model behavior/performance.
\end{abstract}

\section{Hosted file}

Manuscript_Nguyen_et_al.docx available at https://authorea.com/users/420245/articles/526609on-the-evaluation-of-both-spatial-and-temporal-performance-of-distributed-hydrologicalmodels 